\section{Sensory input and circadian effects upon cage activity in two species of cebid monkey*}

\author{
C. STEWART WORTHINGTON† \\ Emory University, Atlanta, Ga. 30322
}

Day and night measures of cage activity in the diurnal squirrel monkey and the nocturnal owl monkey were obtained under various sensory input conditions. Significant activity differences obtained between the species, due to the circadian factor and due to illumination and sound factors. The circadian and illumination variables produced opposite effects from one species to the other. The overall species effect was not significant.

Since the studies of Magoun and associates (1963) demonstrated the existence of sensory collaterals to the brainstem reticular formation, it has been assumed that sensory input would elevate neural activity of the reticular system and that this, in turn, would lead to an increase in general locomotor activity. Data supporting such a conclusion have been developed (Isaac \& DeVito, 1958). However, the generality of this conclusion is suspect in that a depression of general activity has been found to accompany sensory input in some cases (Isaac \& Reed, 1961).

Thus far, such discrepant findings have been obtained with visual input as the sensory mode. Further, it has been noted that those animals which respond positively are animals generally considered to be diurnal (squirrel monkeys and rhesus monkeys), while those becoming less active are those generally labeled nocturnal (rats and cats).

The present study examines this relationship using animals somewhat more phylogenetically proximate. The diurnal squirrel monkey (Saimiri) has a nocturnal cousin similar in size and feeding habits. This nocturnal member of the family Cebidae is the douroucoulis (Aotus), more popularly known as the night or owl monkey.

Circadian behavioral differences between these two otherwise similar primates may indicate disparate relationships between their visual and central nervous systems such that we might expect opposite effects of illumination upon behaviors presumed to reflect arousal level. The present study explores this possibility by

*This research was part of a doctoral dissertation submitted to Emory University Graduate School. The study was supported by Grant MH-04539 from the National Institute of Mental Health and was carried out $u$ hile the author was a predoctoral fellow of that institute. The author wishes to thank Dr. Walter Isaac, under whose direction this research was conducted.

tThe author is presently associated with Armstrong State College, Savannah, $\mathrm{Ga}$. 31406 . measuring general activity under various levels of illumination. Broadband "white" noise was included as an independent variable to check upon the effects of sensory input presumed to be relatively independent of the diurnal cycle. Because activity levels might well be linked to the normal daily cycle but differentially for the two species, testing was done on a morning and night basis. SUBJECTS

Five adult members of each species served as Ss. The squirrel monkeys had been used in behavioral experiments for a period of about 4 years. The owl monkeys were given a period of 90 days from arrival at the laboratory for adaptation to laboratory routine and personnel. Both species were then given a month of adaptation to entering the experimental cage and being placed in the isolation chamber.

\section{APPARATUS}

The sound-deadened isolation chamber was $107 \mathrm{~cm}$ wide $\times 107 \mathrm{~cm}$ high $\times 66 \mathrm{~cm}$ front to back. The chamber was divided by a vertical partition. This allowed an experimental cage to be inserted in either side. Centered on the top of the chamber was a high-fidelity sound source (Voice of Music tape recorder, Model 740) and a ventilation exhaust port. A baffle system provided sound portions of the chamber while maintaining their isolation from one another so that light leaks between them were precluded and sound interchange minimized.

Centered over each cage position was a $3 \frac{1}{2} 2$-in.-diam port through which illumination was provided. The incandescent light source was outside the chamber and shone through a Plexiglas and tissue diffusion plate to insure diffuse overall illumination of the cage interior.

The two experimental cages were constructed of $1-\mathrm{cm} \quad(3 / 8-i n$. plywood. A guillotine door formed the front wall, the floor was expanded metal, while the ceiling was of clear Plexiglas. The animals were provided and exhaust-air channels to both with a space $30.5 \mathrm{~cm}$ wide $\times 45.8 \mathrm{~cm}$ high $\times 45.8 \mathrm{~cm}$ deep within each cage. Centered on the long walls, $11.5 \mathrm{~cm}$ above the floor, was a $2.5-\mathrm{cm}$-diam hole through which the photoelectric beam passed.

\section{PROCEDURE}

The colony contained six living cages with two animals per cage. Three adjacent cages held squirrel monkeys, while the other three, also adjacent, held owl monkeys. Illumination in the living cage interiors ranged from $376.7365 \mathrm{~lx}$ ( $35 \mathrm{fc}$ ) to less than would register on the light meter. The colony temperature was regulated between $24^{\circ}$ and $27^{\circ} \mathrm{C}$

The colony illumination was timer controlled. The lights were turned on at 6 a.m. and off at 6 p.m., providing $12 \mathrm{~h}$ of light and $12 \mathrm{~h}$ of dark each day.

Since primates are quite sensitive to any changes in procedure, an invariant routine was maintained from the initiation of the adaptation phase through the completion of data collection.

Cage activity was measured photoelectrically for a period of $1 / 2 \mathrm{~h}$ under the various experimental conditions. The independent variables were three illumination levels, noise or quiet, day or night, and the species. Thus, the experiment contained 12 distinct combinations of independent variables. An owl monkey and a squirrel monkey were paired and run together. The sequence of conditions was selected randomly without replacement for each pair of animals for each replication.

Illumination levels were recorded at the clear top of the experimental cages, with the light meter directed at the source. Thus, these levels represent the maximum illumination available to the animal. Three levels were used: $376.7365 \mathrm{~lx}$ (35 fc), $53.8195 \mathrm{~lx}$ (5 fc), and less than $10.7639 \mathrm{~lx}$ ( $1 \mathrm{fc}$ ). Noise levels were measured inside the experimental cages. The quiet condition registered 50-60 dB and the noise condition, 80-85 dB.

Experimental sessions commenced twice each day, $2 \frac{1}{2} \mathrm{~h}$ after the 6:00 o'clock illumination shifts. Testing ended between 12:30 and 1:00.

Six adaptation replications were required to obtain stable behavior. Four replications for data collection were then completed.

\section{RESULTS}

The activity scores obtained are presented in Table 1 . These data were reduced by analysis of variance. Systematic effects due to replication did not appear, so this factor was not included.

Noise significantly increased the activity of both species ( $F=11.69$, $\mathrm{df}=1 / 88, \mathrm{p}<.01)$. This effect was 
Table 1

Mean Activity Scores: $30-M$ in Trial

\begin{tabular}{|c|c|c|c|c|c|c|c|}
\hline & \multicolumn{2}{|c|}{ Sound } & \multicolumn{3}{|c|}{ Illumination } & \multicolumn{2}{|c|}{ Circadian } \\
\hline & Quiet & Noise & High & Medium & Low & Day & Night \\
\hline $\begin{array}{l}\text { Saimiri } \\
\text { Aotus }\end{array}$ & $\begin{array}{r}450.2 \\
47.6\end{array}$ & $\begin{array}{r}567.5 \\
65.8\end{array}$ & $\begin{array}{r}532.1 \\
27.1\end{array}$ & $\begin{array}{c}545.4 \\
58.1\end{array}$ & $\begin{array}{r}449.0 \\
84.9\end{array}$ & $\begin{array}{r}558.7 \\
25.6\end{array}$ & $\begin{array}{r}469.0 \\
87.8\end{array}$ \\
\hline
\end{tabular}

much greater in the squirrel monkey than in the owl monkey, yielding a significant interaction of Species by Noise $(F=6.25, \mathrm{df}=1 / 88, \mathrm{p}<.01)$.

Illumination had opposite effects upon the activity of the species, as did the circadian factor. Squirrel monkeys were least active in the dark, most active in moderate illumination, and somewhat less active in high illumination than in moderate. The owl monkeys' activity decreased with increased illumination. Thus, a significant Species by Illumination interaction was generated $(F=4.99$, $\mathrm{df}=2 / 88, \mathrm{p}<.01)$.

Squirrel monkeys were more active in the daytime than at night, while the owl monkeys were more active at night. These effects yielded a significant Species by Circadian interaction $\quad(F=16.69, \quad d f=1 / 88$, $\mathrm{p}<.01$ ).

The error term against which the species main effect failed to prove significant was the Ss-within-species variance $(F=4.73, \mathrm{df}=1 / 8, \mathrm{p}>.05)$. Residual variance provided the error term for all other comparisons.

\section{DISCUSSION}

In all experimental situations, the owl monkeys were less responsive than their diurnal cousins. Whether this reflects a substantive species difference remains speculative until further behavioral work is available.

The fact that a Species by Circadian interaction was found to be significant offers a warning. It appears unwise to make species comparisons without considering circadian effects. Further, in view of the dramatic circadian effects demonstrated within species, here and elsewhere (Aschoff, 1963, Farmer, 1961), observers should hesitate to compare studies upon the same species unless the circadian effects are controlled or demonstrably minimal upon the measures involved.

Previous work with noise input (Isaac \& DeVito, 1958; Isaac \& Reed, 1961 ) indicated a weak positive effect, often in interaction with other sensory input variables. The effect shown in the present study is simple and strongly positive. This finding is consistent with the general hypothesis that sensory input will elevate activation.

The Species by Noise interaction proves interesting in the light of comparative speculation. The squirrel monkeys' activity was elevated to a much greater extent by noise input than was the activity of the owl monkey. If the squirrel monkey is more responsive to environmental stimuli than is the owl monkey, we would expect him to exhibit a higher level of overall activation and a greater response to specific activating input (Isaac, 1960). This seems consistent with the present data.

The hypothesis of opposite illumination effects upon activity in the diurnal vs the nocturnal animal (Isaac \& Reed, 1961) is substantiated in the present study. The activity of the squirrel monkeys peaked in medium illumination, then declined slightly in the high illumination. The response curve for the owl monkey is notably linear by comparison.

The effects of circadian and noise factors were in line with expectancies. Illumination produced expected effects upon the activity of the species, elevating the activity of the diurnal species and depressing the activity of the nocturnal species.

\section{REFERENCES}

ASCHOFF, J Comparative physiology: Diurnal rhythms. Annual Review of Physiology, 1963, 25, 581-600.

FARMER, D. S. Photoperiodicity. Annual Review of Physiology, 1961, 23, $71-96$.

ISAAC, W. Arousal and reaction time in cats. Journal of Comparative \& Physiological Psychology, 1960, 53, 234-236

ISAAC, W.. \& DeVITO, J. Effect of sensory stimulation on the activity of normal and prefrontal lobectomized monkeys. Journal of Comparative \& Physiological Psychology, 1958, 51, 172-174.

ISAAC, W. \& REED, W. G. The effect of sensory stimulation on the activity of cats. Journal of Comparative \& Physiological Psychology, 1961, 54, $677-678$.

MAGOUN. H. W. The waking brain. (2nd ed.) Springfieid, Ill: Thomas, 1963. 